University of Nebraska - Lincoln

DigitalCommons@University of Nebraska - Lincoln

\title{
Impact damage on a thin glass plate with a thin polycarbonate backing
}

Wenke Hu

University of Nebraska-Lincoln

Yenan Wang

University of Nebraska-Lincoln

Jian Yu

U.S. Army Research Laboratory, jian.h.yu@arl.army.mil

Chian-Fong Yen

U.S. Army Research Laboratory

Florin Bobaru Ph.D.

University of Nebraska-Lincoln, fbobaru2@unl.edu

Follow this and additional works at: https://digitalcommons.unl.edu/mechengfacpub

Hu, Wenke; Wang, Yenan; Yu, Jian; Yen, Chian-Fong; and Bobaru, Florin Ph.D., "Impact damage on a thin glass plate with a thin polycarbonate backing" (2013). Mechanical \& Materials Engineering Faculty Publications. 92.

https://digitalcommons.unl.edu/mechengfacpub/92

This Article is brought to you for free and open access by the Mechanical \& Materials Engineering, Department of at DigitalCommons@University of Nebraska - Lincoln. It has been accepted for inclusion in Mechanical \& Materials Engineering Faculty Publications by an authorized administrator of DigitalCommons@University of Nebraska Lincoln. 


\title{
Impact damage on a thin glass plate with a thin polycarbonate backing
}

\author{
Wenke $\mathrm{Hu}^{\mathrm{a}}$, Yenan Wang ${ }^{\mathrm{a}}$, Jian $\mathrm{Yu}^{\mathrm{b}}$, Chian-Fong Yen ${ }^{\mathrm{b}}$, Florin Bobaru ${ }^{\mathrm{a}, *}$ \\ ${ }^{a}$ Department of Mechanical and Materials Engineering, University of Nebraska-Lincoln, Lincoln, NE 68588, United States \\ ${ }^{\mathrm{b}}$ U.S. Army Research Laboratory, Aberdeen Proving Grounds, MD 21005, United States
}

\section{A R T I C L E I N F O}

\section{Article history:}

Received 5 September 2012

Received in revised form

27 June 2013

Accepted 1 July 2013

Available online 13 July 2013

\section{Keywords:}

Glass

Multi-layer

Brittle fracture

Impact

Peridynamics

\begin{abstract}
A B S T R A C T
We present experimental and computational results for the impact of a spherical projectile on a thin glass plate with a thin polycarbonate backing plate, restrained in a metal frame, or in the absence of the frame. We analyze the dependence of the damage patterns in the glass plate on the increasing impact velocities, from $61 \mathrm{~m} / \mathrm{s}$ to $200 \mathrm{~m} / \mathrm{s}$. Experimental results are compared with those from peridynamic simulations of a simplified model. The main fracture patterns observed experimentally are captured by the peridynamic model for each of the three projectile velocities tested. More accurate implementation of the actual boundary conditions present in the experiments will likely further improve modeling of brittle damage from impact on a multi-layered system. The peridynamic computational model sheds light into the early stages of the complex brittle damage evolution in the glass layer, and the influence of boundary conditions on the dynamic fracture process.
\end{abstract}

(c) 2013 Elsevier Ltd. All rights reserved.

\section{Introduction}

Due to their low cost of manufacturing, light weight, and high performance, glass laminates are widely used in skylight glazing and in windshields for automobile, train, and military vehicle. In order to design and improve glass laminates for protective applications, it is important to be able to predict the damage and fracture behavior of glass plates under impact.

Experiments have been conducted in the past (see Refs. [1-8]) to analyze the behavior of a single glass plate or glass laminates subject to low or high impact velocity. Knight et al. [1] found that the Hertzian cone angle decreases with increasing impact velocity for a glass block under small steel spheres impact. Ball and Mckenzie [2] performed a series of impact tests on circular float glass plates with thicknesses between $3 \mathrm{~mm}$ and $12 \mathrm{~mm}$ with various impact speed $(10-50 \mathrm{~m} / \mathrm{s})$. They identified a number of failure mechanisms in the plates and constructed a fracture map which incorporates the effect of plate thickness and impact velocity. Important contributions to experimental analysis of damage in glass from impact and understanding the mechanisms of brittle failure from solid particle impact have been made over several decades by the group at Cavendish laboratory at University of Cambridge (see Ref. [3]). For example, Field ([4,5]) and Field and

\footnotetext{
* Corresponding author.

E-mail address: fbobaru2@unl.edu (F. Bobaru).
}

Sun [6] studied the impact of small steel spheres on a range of glasses and ceramics. They give tables of the threshold velocities at which different damage mechanisms operate. For instance, in glasses, single cone cracks can be formed up to $40 \mathrm{~m} / \mathrm{s}$, while at higher impact speeds, stress wave damage rather than a 'quasistatic' response is obtained. Their research ([4-6]) also identified important differences in the response depending on whether the ratio of the hardness of the projectile to the target was greater or less than 1.The thickness of the glass plate in relation to the impact velocity, and the angle of impact are also important factors affecting the progression of damage in glass (see Refs. [1,7]). The picture of damage progression is further complicated in multi-layered systems due to the stress wave reflections from the various interfaces. Multi-layer glass laminates subject to high velocity impact display very complex damage pattern by dynamic brittle fracture through the layers. Bless et al. [8] studied the morphology of damage from high velocity impact $(1120 \mathrm{~m} / \mathrm{s})$ onto a seven-layer glass laminate with a polycarbonate backing.

Developing models that can correctly capture all the observed features of dynamic fracture and fragmentation from impact on glass plates and laminates has been extremely challenging. A number of numerical studies based on the finite element method (FEM) or molecular dynamics [9-12] have been performed to compute the response of glass plates and laminates under impact loading. In the work of Timmel et al. ([9]), glass laminates with a PVB-interlayer under low impact velocity are modeled using LSDYNA shell elements and a failure criterion based on the 
principal strain reaching a critical value. Element deletion is used as the elements fail. The fracture behavior is highly mesh dependent and certain meshes lead to unrealistic results. Finite elements with element deletion method (sometimes called "vanishing element technique" or "element erosion method") are also used in Refs. [10], with a stress-based continuum damage model for describing the constitutive behavior of glass. The contribution of different cracking systems is explicitly taken into account in this work. The authors of [10] study the initiation and propagation of the ring/cone crack in a glass plate under impact based on this model. They obtained multi-ring cracks when using a higher critical stress value in contrast with extensive crushing and ejected debris when the critical stress value used was lower. Since a 2D axisymmetric model is used in Refs. [10], only a limited set of cracks are obtained: for instance, radial cracks cannot be simulated with this approach.

Motivated by experimental observation of glass failure, the authors of reference [11] proposed a model based on the idea that a critical energy value must be reached over a finite region before the glass can fail, independent of the calculated stress. This is a nonlocal damage model. A finite element model using shell elements implements this nonlocal criterion for a compound glass-PVB-glass system and uses element deletion once failure is determined to occur. While the nonlocal damage model improves the simulation results compared with local damage models, the obtained damage morphology differs in significant ways with the experimentally observed one.

At the other end of the spectrum in terms of modeling failure of glass, atomistic models have been used recently (see e.g Ref. [12].) to study the fracture of silica glass from hypervelocity impact. The authors of [12] report cracks initiate and propagate from the bottom to the top of the 24 nano-meter thick glass sheet. Molecular dynamics (MD) models, however, cannot be used to model macroscale structures, while multiscale models coupling MD with FEM models, for example, need to take into account the well-known fact (see e.g Ref. [13].) that stress waves that propagate and reflect from the boundaries strongly affect the evolution of fracture in a brittle system. Therefore, to accurately capture the evolution of damage in brittle systems, the entire structure needs to be considered in the simulations and the dynamics of the stress waves, in the presence of evolving damage and failure fronts, needs to be solved for correctly.

In the present paper, we report on results from experimental and numerical studies using peridynamics on impact on a twolayer square laminate composed of a thin $(33 \mathrm{~mm})$ glass plate and a thin $(30 \mathrm{~mm})$ polycarbonate backing plate with sides of about $10 \mathrm{~cm}$. The two-layer system is placed in a metallic frame and impacted with a spherical projectile at speeds ranging from $61 \mathrm{~m} / \mathrm{s}$ to $200 \mathrm{~m} / \mathrm{s}$. We will refer to this glass-polycarbonate system as the $\mathrm{G}-\mathrm{PC}$ system. The numerical studies conducted with peridynamics, using the code EMU (see Ref. [14]) with some modifications of the spatial integration detailed in Ref. [15] are performed for a model which only approximates the presence of the actual aluminum frame used in the experiments. The recently introduced nonlocal continuum model, peridynamics [16], is well suited for solving dynamic fracture problems (see e.g Refs. [17-22].). In order to overcome mathematical inconsistencies in the classical continuum mechanics models of problems in which cracks are initiated and evolve in time, peridynamics uses an integral of forces (per volumesquared) over a nonlocal region (called horizon) around a point to replace the divergence of the stress tensor in the equations of motion. The method has been shown to correctly predict phenomena in dynamic fracture in brittle materials and composite materials, including crack branching and the crack propagation speed [16-20].The role of the "influence function" on the peridynamic results for impact damage on a glass cylinder from a spherical projectile has been studied in Ref. [23].

Here, we employ the simplest peridynamic constitutive model for a brittle material (linear micro-elasticity with brittle damage) and observe that we capture most of the damage morphology seen in the experiments, including the changes induced by the higher impact speed on the damage patterns. Furthermore, the trend for the projectile rebound speed compares favorably with the experimental results. The boundary conditions used in the simulations are for a more rigid setting than those present in the experiments; most of the differences between the computational results and the experimental observations can be attributed to the rigidity of the boundary conditions used here to approximate the holding metal frame employed in the experiments. The evolution of damage and its connection to the stress waves' propagation in the two-layer system is described in detail.

The paper is organized as follows: in Section 2, the experimental tests are described and the investigation of damage patterns dependence on the impact speeds is presented; in Section 3, we briefly review the basic formulation for peridynamics and the damage model used; in Section 4, we present the numerical results from the peridynamic model of the impact on the G-PC system and compare them with the experimental results. We also include a description of the early stages of damage evolution in the glass layer, based on the computational model results. Conclusions are given in Section 5.

\section{Experiments of impact on the glass-polycarbonate system}

\subsection{Experimental setup}

The two layer glass-polycarbonate (G-PC) system tested at the ARL lab consists of a square soda-lime glass plate with dimensions $10.16 \mathrm{~cm} \times 10.16 \mathrm{~cm} \times 0.33 \mathrm{~cm}$, and a backing polycarbonate layer of $10.16 \mathrm{~cm} \times 10.16 \mathrm{~cm} \times 0.3 \mathrm{~cm}$. An aluminum frame holds the GPC plates together and has the same outer dimensions as the G-PC laminate, but has an opening of $5.08 \mathrm{~cm} \times 5.08 \mathrm{~cm}$ (see Fig. 1). The inside surface of the frame is lined with a $1 \mathrm{~mm}$ thick rubber gasket. Tests were also performed without rubber gasket but no major differences are observed between these two conditions in terms of damage patterns. The frame, along with the G-PC system, is clamped on the edge to a target fixture for impact testing. A spherical projectile (440C stainless steel) with $0.556 \mathrm{~cm}$ diameter and $0.692 \mathrm{~g}$ mass is shot from a gas gun normal to the center of the glass plate at different velocities: 61, 100, and $200 \mathrm{~m} / \mathrm{s}$.

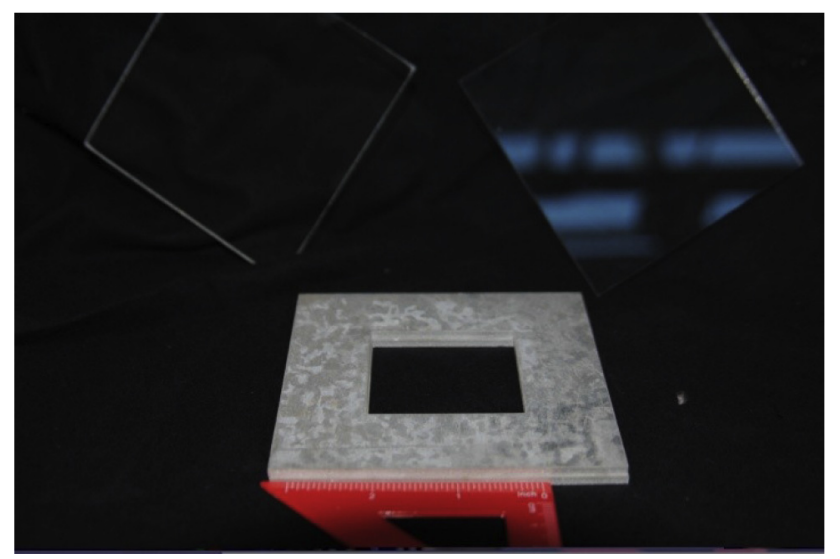

Fig. 1. Experimental setup. The glass and polycarbonate plates are sandwiched between two metal frames and clamped together at the outer edge. 


\subsection{Experimental results and discussion}

Fig. 2 shows the post-mortem samples (glass plate only) impacted at three different velocities. The polycarbonate plate is not damaged at the lower two impact velocities and at $200 \mathrm{~m} / \mathrm{s}$ only some minor plastic deformation is observed near the impact. The signature of the metal frame opening is visible from the cracks enclosed in the rectangular regions marked on the figures in each of the three impact speed cases. Note that clamping is not at the opening's edges but on the outer edges of the frame, thus some separation between the glass and PC layers, and the metal frame is possible and likely takes place during the impact. This possible separation explains the non-uniformity of the cracks in the frame opening area. Radial cracks emanating from the impact crater are observed in all cases, but with some differences in terms of the length and number of these radial cracks, which depend on the impact velocity. Circumferential cracks or ripple cracks (see elliptical regions in Fig. 2) become denser as the impact speeds increase. Some of the through-thickness cracks are not perpendicular to the plane of the plates, instead they are tilted, as they can be seen from the light reflections in the diamond regions. For impact speeds of $100 \mathrm{~m} / \mathrm{s}$ and $200 \mathrm{~m} / \mathrm{s}$, some of the radial cracks split (or branch) as seen inside the circular regions. If these cracks form from the edges, however, then they are coalescing, instead of branching. These features seem to associate primarily with cracks that split the plate into four quadrants, which are called quadrant cracks in Ref. [8]. In the $100 \mathrm{~m} / \mathrm{s}$ case, we also observe the formation of cracks that do not connect to the central region, thus they could grow from the boundaries, as seen in the triangular regions. These cracks are harder to distinguish in the $200 \mathrm{~m} / \mathrm{s}$ case due to the severe fragmentation that had taken place. Corner cracks cutting the corners at $45^{\circ}$ (see square regions) from the impact velocity of $200 \mathrm{~m} / \mathrm{s}$ at three of the four corners. These types of cracks are absent at impact speeds of $61 \mathrm{~m} / \mathrm{s}$ and $100 \mathrm{~m} / \mathrm{s}$.

At the two low impact speeds $(61 \mathrm{~m} / \mathrm{s}$ and $100 \mathrm{~m} / \mathrm{s})$, no visible damage is observed in the polycarbonate plate, aside from some superficial surface scratches. At these impact speeds, the projectile rebounds from the target with speeds of about $3 \mathrm{~m} / \mathrm{s}$ and $8 \mathrm{~m} / \mathrm{s}$, respectively. At a high impact speed of $200 \mathrm{~m} / \mathrm{s}$, plastic deformation near the impact site is observed in the polycarbonate plate but no cracks or penetration. At this impact speed the projectile rebounds from the G-PC system faster than the rebound from the two other two impact speeds, at about $33 \mathrm{~m} / \mathrm{s}$.

Some glass fragments around the impact crater were lost during the impact on the strike face. Tape was used to hold the pieces together after the tests. At the highest impact speed, the predominant damage pattern outside of the central region, where the fine and dense radial and circumferential cracks are formed, is

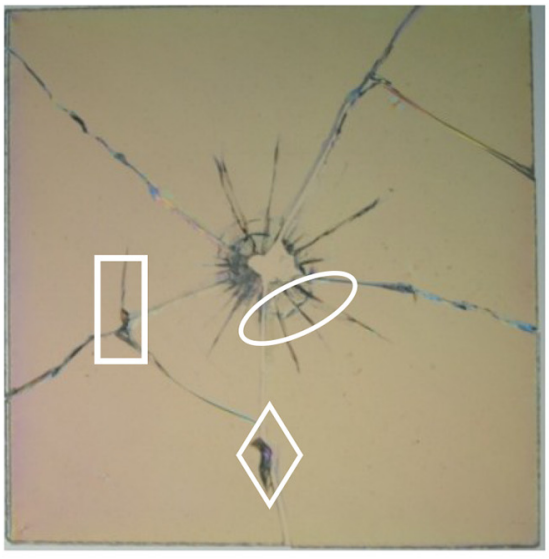

$[61 \mathrm{~m} / \mathrm{s}]$

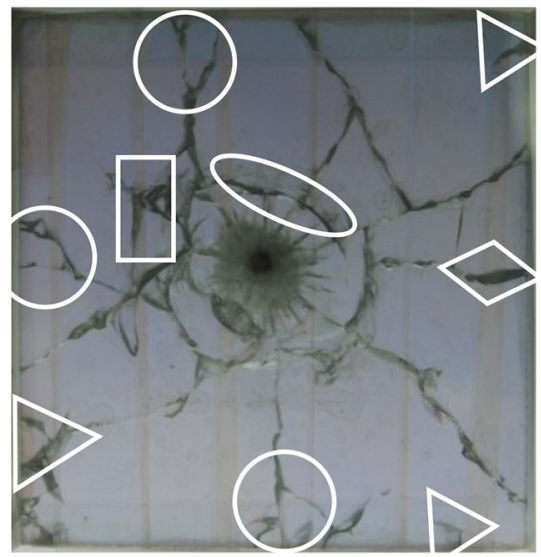

$[100 \mathrm{~m} / \mathrm{s}]$

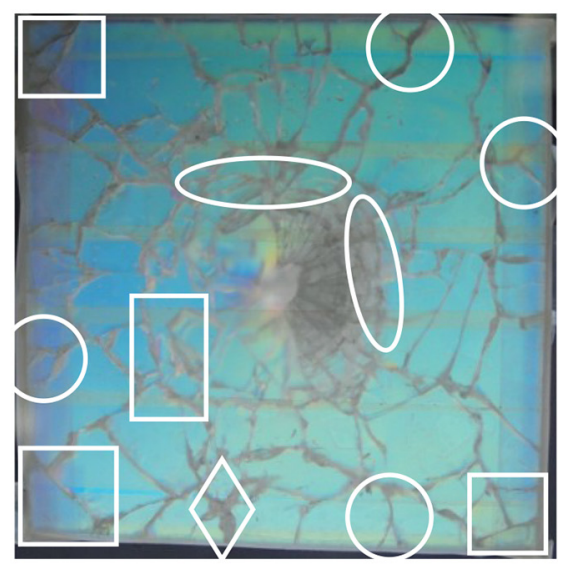

$[200 \mathrm{~m} / \mathrm{s}]$

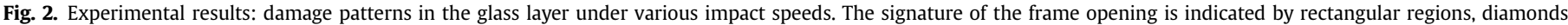

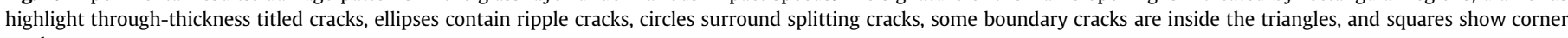
cracks. 
fragments of glass that have some resemblance to intersecting radial and circumferential cracks. However, in this case, the "radial" and "circumferential" cracks are discontinuous and not well organized, the picture showing mostly irregular "islands" of unbroken glass. Cracks that split near the boundaries, or that coalesce if they are actually starting from the boundaries, are observed as well on each of the four sides. A unique feature observed at the high impact speed and not seen at 61 or $100 \mathrm{~m} / \mathrm{s}$, is the presence of corner cracks.

\section{Review of the peridynamic formulation}

Introduced by Silling [16], the peridynamic theory is a non-local formulation that extends the classical continuum mechanics formulation. The term "peridynamics" comes from the Greek roots for "nearby" and "force" [16]. In peridynamics, every material point is connected to the other points inside a certain "horizon" region through peridynamic bonds. Instead of the stress divergence term in the classical equations of motion for a continuum, an integral of forces per volume-squared in the bonds at a point, over the current point horizon region is used. The peridynamic equations of motion are:

$\rho \ddot{\mathbf{u}}(\mathbf{x}, t)=\int_{H} \mathbf{f}(\mathbf{u}(\mathbf{x}, t)-\mathbf{u}(\mathbf{x}, \mathrm{t}), \widehat{\mathbf{x}}-\mathbf{x}) \mathrm{dV}_{\widehat{\mathbf{x}}}+\mathbf{b}(\mathbf{x}, t)$

where $\mathbf{f}$ is the pairwise force function in the peridynamic bond that connects point $\hat{x}$ to $\mathbf{x}$, and $\mathbf{u}$ is the displacement vector field. $\rho$ is the density and $\mathbf{b}(\mathbf{x}, t)$ is the body force. The integral is defined over a region $H$, the compact supported domain of the pairwise force function around a point $\mathbf{x}$, called the "horizon region". The region is taken here to be a sphere of radius $\delta$. The peridynamic horizon may be viewed as an "effective" interaction distance or an "effective length-scale" of a continuum [21]. In principle, the exact size and shape of the horizon could be found from wave dispersion curves for a specific material under specific dynamic conditions (see Refs. [16,24]).

Let $\xi=\widehat{\mathbf{x}}-\mathbf{x}$ be the relative position in the reference configuration and $\eta=\widehat{\mathbf{u}}-\mathbf{u}$ the relative displacement between two material points, $\mathbf{x}$ and $\widehat{x}$. From the definition of the horizon, we have

$\|\xi\|>\delta \Rightarrow \mathbf{f}(\eta, \xi)=0$

A micro-elastic material is defined as one for which the pairwise force derives from a micropotential $\omega$ :

$\mathbf{f}(\eta, \xi)=\frac{\partial \omega(\eta, \xi)}{\partial \eta}$

The strain energy density at a given point is defined as:

$W=\frac{1}{2} \int_{H} \omega(\eta, \xi) \mathrm{dV}_{\xi}$

The factor $1 / 2$ factor is present because the elastic energy in a bond is shared by the two nodes connected by the bond. A linear micro-elastic potential, which leads to a linear relationship between the bond force and the relative elongation of the bond, is obtained if we take

$\omega(\eta, \xi)=\frac{c(\|\xi\|) s^{2}\|\xi\|}{2}$

where $s$ is the bond relative elongation $s=\frac{\|\xi+\eta\|-\|\xi\|}{\|\xi\|}$

The corresponding pairwise force becomes

$\mathbf{f}(\eta, \xi)=\frac{\partial \omega(\eta, \xi)}{\partial \eta}=c(\|\xi\|) s \frac{\partial\|\xi+\eta\|}{\partial \eta}$

The function $c(\|\xi\|)$ is called the micromodulus function and represents the bond elastic stiffness. For isotropic materials, the micromodulus function version is computed by matching the peridynamic strain energy density to the classical strain energy density

$W_{\text {classical }}=\frac{1}{2} \int_{H} \frac{c(\|\xi\|) s^{2}\|\xi\|}{2} \mathrm{dV}_{\widehat{x}}$

The value for the 3D constant micromodulus function is then derived as (see Ref. [25])

$c=\frac{18 k}{\pi \delta^{4}}$

where $k$ is the bulk modulus.

The damage model in peridynamics consists in breaking the peridynamic bonds connecting any two nodes when the relative change in distance between them exceeds a certain prescribed value $s_{0}$ [16]. This critical relative elongation parameter $s_{0}$ is obtained by equating the work, per unit fractured area, required to break all the bonds across a surface, to the fracture energy required for complete separation along the surface (see Ref. [25])

$G_{0}=\int_{0}^{\delta} \int_{0}^{2 \pi} \int_{\mathrm{z}}^{\delta} \int_{0}^{\cos ^{-1}(z /\|\xi\|)}\left[c(\|\xi\|) s_{0}^{2}\|\xi\| / 2\right]\|\xi\|^{2} \sin \varphi \mathrm{d} \varphi \mathrm{d} \xi \mathrm{d} \theta \mathrm{d} z$

For the constant micro-modulus given above, the critical relative elongation is obtained as

$s_{0}=\sqrt{\frac{10 G_{0}}{\pi c \delta^{5}}}$

We impose the same $s_{0}$ over the entire region, which results in an effectively slightly weaker material in the regions near the boundary or where damage already happened, since the integration region is reduced compared to a location in the undamaged bulk. This is similar to the skin effect discussed in Ref. [15]. A damage-dependent $s_{0}$ can be introduced in order to reduce the effectively weaker bond strength near the boundary or in damaged regions (see Refs. $[25,18]$ ). Especially in impact problems where many new boundaries are formed as a result of a multitude of newly created fracture surfaces, a constant critical relative elongation may result in the over prediction of the number of fragments formed. In this paper we choose to "strengthen" the material in damaged regions by allowing $s_{0}$ to depend monotonically on the amount of damage at that particular node. One could attempt an exact evaluation of the damage-dependent (variable) $s_{0}$. However, here we resort to a simpler way, namely an approximation proposed in Ref. [14] which is described by the following formula

$s=\left\{\begin{array}{cc}s_{0} \times \min \left[\gamma, 1+\beta \times \frac{D-\alpha}{1-D}\right], & \text { if } D>\alpha \\ s_{0}, & \text { otherwise }\end{array}\right.$

where the damage index $D$ is defined as the ratio of the number of broken bonds to the number of initially bonds. $s_{0}$ and $s$ are the initially given critical relative elongation and the computed critical 
relative elongation, respectively. In the simulations that follow, we use the values $\alpha=0.35, \beta=1$, and $\gamma=2$. More details can be found in Ref. [18].

A validation of the peridynamic micro-elastic model against a finite element model has been performed in Refs. [22], where a comparison of the elastic bending deformation between the nonlocal solution for the problem of elastic impact, in which no damage is allowed to initiate, with the horizon size of about $2.7 \mathrm{~mm}$ and a grid spacing less than $1 \mathrm{~mm}$, and a dynamic (explicit) finite element solution obtained by using Abaqus. The results for the bending deformation in the problem of elastic impact of a spherical rigid projectile onto a two-layer glass backed by a polycarbonate layer given by a converged finite element solution with solid elements and the peridynamic model that uses a grid of comparable size were shown to match very well. The time-evolution for the projectile speed also matches well between the two different models (see Ref. [22]).

For the model with damage, the comparison of the peridynamic results will be made with the experimental results discussed in Section 2 .

\section{Peridynamic results for impact on the framed thin-plate glass-polycarbonate system}

\subsection{Problem setup}

The peridynamic model for the two layer G-PC system uses the geometry and boundary conditions shown in Fig. 3. The geometrical dimensions are the same as in the experiments, but the boundary conditions are slightly different from those present in the experiments. For example, in the experiment, two metal frames with clamps are used in order to hold the specimen. Since a model that includes the metal plate, the clamps, and the holding fixture would lead to a much larger computational simulation, and since the exact pressure applied on the clamps is not known with precision, we choose here to approximate the actual boundary conditions with the following: zero out-of-plane displacements $(z-$ direction in Fig. 3) for the nodes on the top face of the glass plate and bottom face of the polycarbonate layer, over the frame region (see Fig. 4). We expect such conditions to lead to cracking along the inner boundary of the frame due to prevention of out-of-plane bending, but this issue will be analyzed in detail in Section 5 where we conduct tests with almost no boundary conditions.

In the experimental samples, there is no adhesive layer between the glass and the polycarbonate. In the computational model, we "disconnect" all the peridynamic bonds between the two different material regions (glass and polycarbonate) and use simple nonpenetrating contact conditions to describe contact conditions.

The material properties used in the model for soda-lime glass are: density $\rho=2440 \mathrm{~kg} / \mathrm{m}^{3}$, Young's modulus $E=72 \mathrm{GPa}$, facture energy $G_{0}=8 \mathrm{~J} / \mathrm{m}^{2}$. In the experimental results, no damage is observed in the polycarbonate layer, except for the highest impact

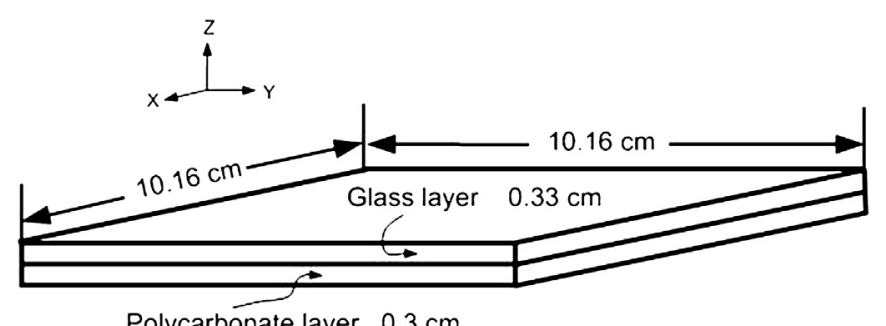

Polycarbonate layer $0.3 \mathrm{~cm}$

Fig. 3. Dimensions for the two-layered glass-polycarbonate system.

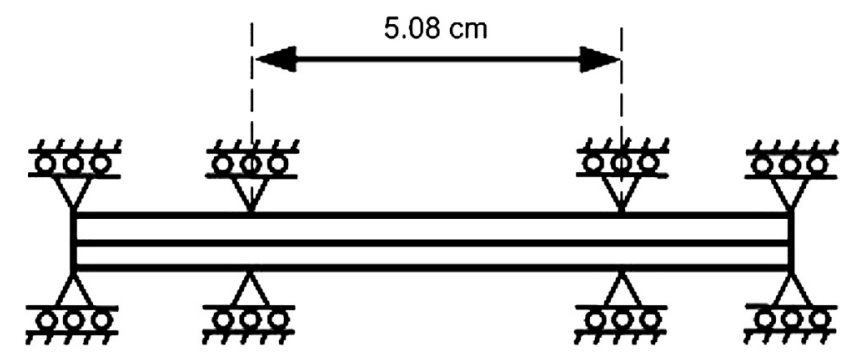

Fig. 4. Through-thickness view (along the $x-z$ plane passing through the center of the plate) of the boundary conditions for the glass-polycarbonate system.

speed where some plastic deformation is visible. As a consequence, in the peridynamic simulations of the polycarbonate layer we prescribe "no-fail" conditions, by using a sufficiently large critical relative elongation that is never met during the simulation. The material properties for the polycarbonate layer are: density $\rho=1200 \mathrm{~kg} / \mathrm{m}^{3}$, Young's modulus $E=2 \mathrm{GPa}$. In bond-based peridynamics, the version used in this paper, the Poisson's ratio is fixed and equals 0.25 in 3D (close to the soda-lime glass Poisson ratio of 0.22 ), and $1 / 3$ in $2 \mathrm{D}$, since the assumption is that material points interact only through a pair-potential. The Poisson ratio limitation is removed in the state-based formulation of peridynamics (see Ref. [26]), however, here we use the bond-based for simplicity. We note that, in dynamic fracture problems of the type considered in this work, the Poisson ratio value does not have a significant influence on the crack propagation speed or the shape of the crack path (see Refs. [17,26]).

In the simulations, we use a spherical rigid projectile of the same dimensions and mass as the steel projectile used in the experiments and described in Section 2. The projectile is placed very close to the glass plate so that impact happens in the first few time steps. The use of a deformable projectile is left for the future.

We perform the simulations using the same impact speeds as those used in the experiments. Based on a convergence study performed in Refs. [27], we select to use a horizon size $\delta=1 \mathrm{~mm}$ and $m=\delta / \Delta x=4$, where $\Delta \mathrm{x}$ is the grid spacing used for the discretization of the domain integral in the peridynamics equations of motion. The selection is based on the damage patterns results and the projectile speed after perforation of a single glass plate (see Ref. [27]). Discussions about the dependence or independence of the crack propagation speed on the horizon size are given in Refs. [21] and [22]. The 3D discrete model has a total of over 6 million nodes (about 20 million degrees of freedom). The total simulation time is $77 \mu \mathrm{s}$ with a time step size of about $27.5 \mathrm{~ns}$. We use 100 processors on $2.2 \mathrm{GHz} / 64$ bit Opteron Linux cluster with 70 Gb memory.

\subsection{Damage maps for different impact velocity: results and discussions}

The peridynamic results for the damage of the glass plate are presented in terms of nodal damage index maps. The damage index $d$ at a node is defined as:

$d=\frac{n_{\text {broken }}}{n}$

where $n_{\text {broken }}$ and $n$ are the number of broken bonds and the number of initial bonds at a node, respectively. The damage index is a number between 0 and 1 ( 0 means no bonds connected at the node are broken, and 1 means all the bonds are broken). Note that a damage index of around $0.4-0.5$ may indicate that a fracture surface exists, if damage is localized along a surface (line). Diffuse 
damage regions may indicate a "shattered" zone or an area of densely packed cracks that are too fine to resolve with a certain discretization/horizon (see Ref. [20]). The legend shown in Fig. 5 is used in all damage plots.

In Figs. $5-7$, the damage maps for the top face ( $x-y$ plane), bottom face $(x-y$ plane), and cross-sectional cut ( $x-z$ plane) through the center of the glass plate are shown at about $77 \mu \mathrm{s}$. We compare our simulation results with experimental results for the three impact speeds, 61, 100, and $200 \mathrm{~m} / \mathrm{s}$.

\subsubsection{Results for impact speed of $61 \mathrm{~m} / \mathrm{s}$}

From Fig. 5 we observe that radial cracks extend from the central crater region along the quadrant directions (towards the middle of the sides of the square plate) and the diagonal directions (towards the plate corners). For the region nearby the crater, radial cracks appear to be straight (see damage on the bottom face in Fig. 5(b)), and the diagonal cracks continue to be straight whereas the quadrant cracks have more turns. These features can also be distinguished in the experimental result in Fig. 5(d). Circumferential or ripple cracks (see the highlighted elliptical areas) form in the computational model at about the same distance from the center as in the experiments. The mark left by the frame opening is much more prominent in the peridynamic simulations than in the experiment (see the highlighted rectangular areas) because in the computations we impose rigid, zero displacements conditions over where the metal frames are in the experimental setup. The actual aluminum frame deforms slightly (in bending) as the G-PC system bends due to impact. The imperfect contact between the frame's inner boundary and the glass leads to some cracking. The damage maps also indicate another remarkable characteristic of fracture induced by impact on the G-PC system: in the diamond-shaped zones, a quadrant radial crack kinks just before reaching the boundary and the photograph of the experiment shows that the through-thickness crack is not perpendicular onto the plane of the plate, but it is rather tilted. A similar pattern is displayed by the peridynamic results, in which two quadrant cracks kink before reaching the boundary and the slight difference between the damage paths on the strike face and the back face of the glass plate means that these cracks are tilted through the thickness. Stress waves propagating through the G-PC system are responsible for these features.

By examining the cross-sectional view of the peridynamics solution in Fig. 5(c), we observe a Hertz-type cone crack transitioning into a transverse crack (parallel to the strike face) form in sequence as a result of the impact and wave reflections from the polycarbonate backing plate.

The time-evolution of the system of cracks for this impact velocity is discussed in Section 4.4.

\subsubsection{Results for impact speed of $100 \mathrm{~m} / \mathrm{s}$}

At the impact speed of $100 \mathrm{~m} / \mathrm{s}$, the mark of the frame opening is now stronger, with cracks showing on both the strike and the back face of the glass plate (see Fig. 6). As before, due to the more compliant response of the actual metal frame than the rigidly imposed boundary in our model, the experimental results indicate the presence of the frame opening in a much reduced way (see highlighted rectangular regions). Ripple cracks (marked by ellipses) now show farther away from the center of the plate than with the low impact speeds and they are not continuous but appear to be interrupted by radial cracks. The distinct difference between the

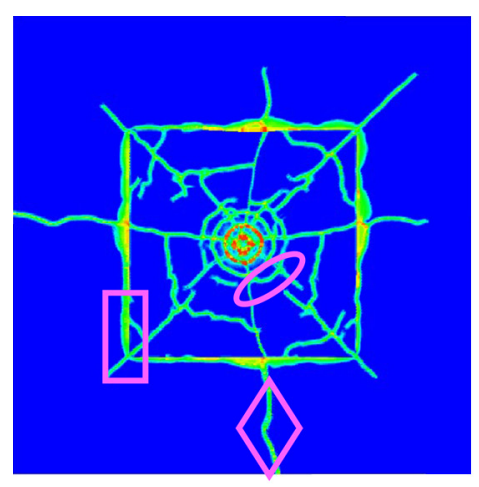

(a)

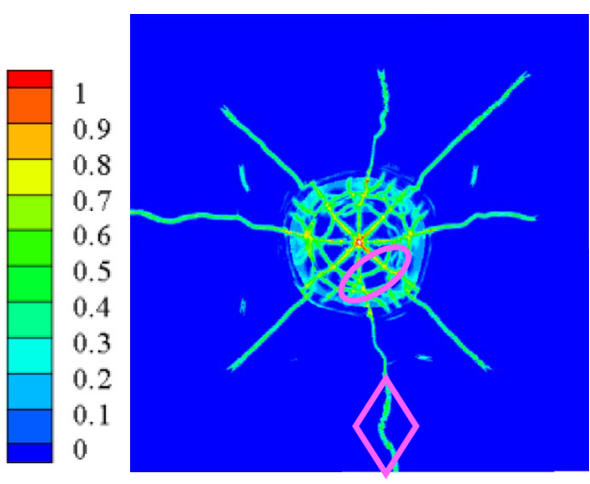

(b)

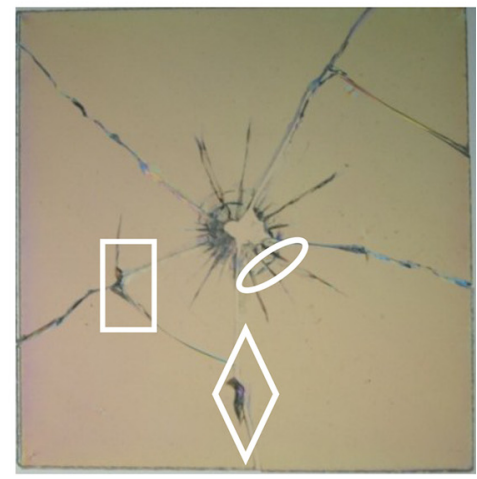

(d)

(c)

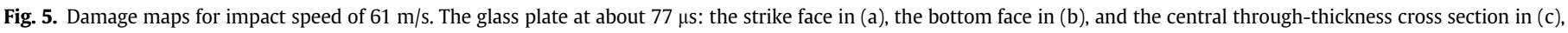
where displacements are enhanced by a factor of two, to better observe separation, and thickness direction is enlarged; experimental result shown in (d) 


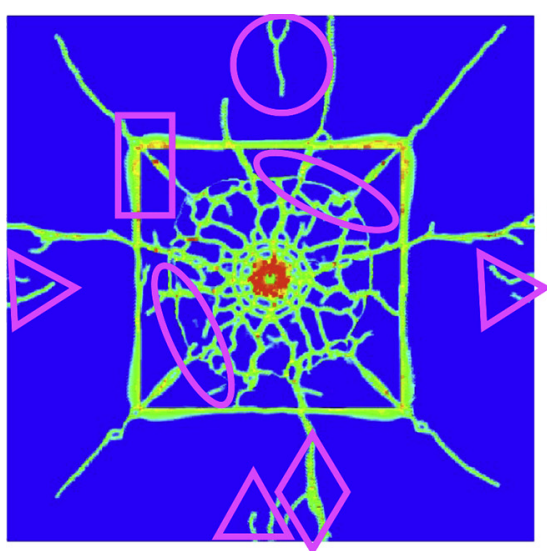

(a)

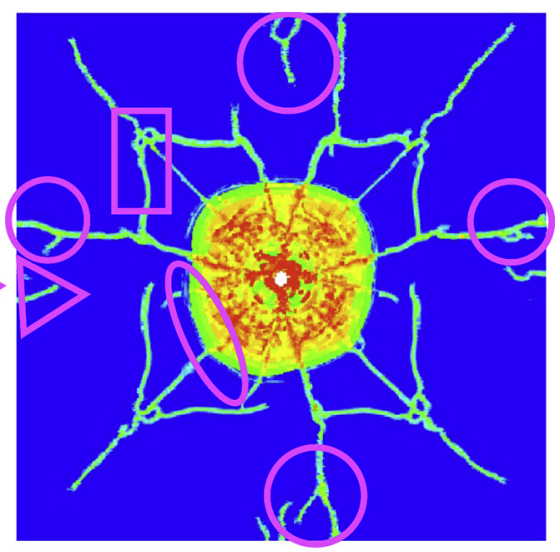

(b)

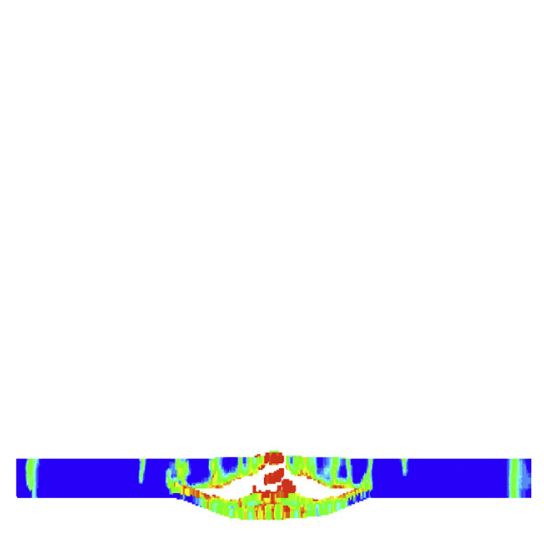

(c)

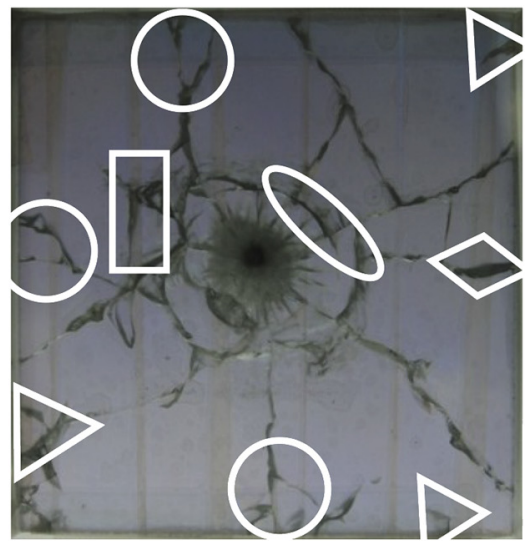

(d)

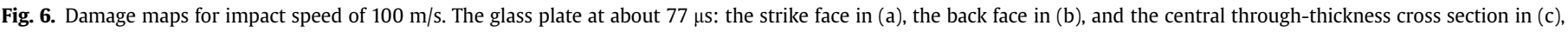
where displacements are enhanced by a factor of two, to better observe separation, and thickness direction is enlarged; experimental result shown in (d).

damage on the strike face and back face of the glass plate near the center of the plate can be better understood from the crosssectional view in Fig. 6(c). It is interesting to remark that the blurry region, between the center of the plate and the first major ripple crack observed in the experimental results in Fig. 6(d), is an indication of the conical crack we observed in the simulations (Fig. 6(c)). The back face damage is extensive and diffuse damage (shattered glass) whereas on the front face, outside of the crater region, localized damage develops in the form of crack lines. Some cracks appear to start from the boundary of the structure in both the experiments and simulations (see zones marked by triangles). The quadrant cracks branch out (see regions marked by circles) as they approach the boundaries. In most cases, the corresponding crack paths on the strike face and back face of the glass plate are not identical, demonstrating that such cracks have tortuous throughthickness surfaces. This is observed in the experiments as well.

This particular feature is seen in the photograph of the postmortem sample as a darker and thicker crack line, due to the light reflection (see Fig. 6(d)).

\subsubsection{Results for impact speed of $200 \mathrm{~m} / \mathrm{s}$}

When the G-PC system is subject to impact speed of $200 \mathrm{~m} / \mathrm{s}$, the damage obtained by the peridynamic model is extensive and most of the glass layer is fragmented. Three different regions can be identified from Fig. 7 (a) and (b): a central region with finely spaced radial cracks on which a few ripple cracks superpose, a region that can be considered as the signature of the boundary conditions that mimic the frame opening, and the rest of the plate in which mostly large and some small size fragments are formed. These regions seen from the experiments are well captured by the peridynamic simulation, except for the signature cracks related to the frame opening: it is quite possible that under such impact energy, there is partial separation between the holding frame and the glasspolycarbonate plates and due to this, the frame opening signature is hardly visible (see the highlighted rectangular area in Fig. 7(d)) at this impact speed. In the simulations, considerable amount of damage is seen around the opening area and this is attributable to the rigid boundary conditions imposed there. The difference between the damage seen on the strike face and the back face in this area is due to the presence of the backing polycarbonate plate that is in contact with the back face of the glass layer, whereas the top face of the glass layer has the imposed zero-displacements outside of the frame opening area. Since there is no energy dissipation mechanism in the model other than via breaking of bonds, stress waves create more damage than in the experiment where some of the energy is transferred to the metal frame and the holding fixture. Notice also that through-thickness corner cracks (highlighted square regions) are a new feature seen in the experiments, not observed at the lower impact speed. The peridynamic simulations capture the corner cracks very well. For these corner cracks, the damage maps of the strike and back faces are similar, meaning that these cracks are straight through-thickness cracks, and not tilted. 


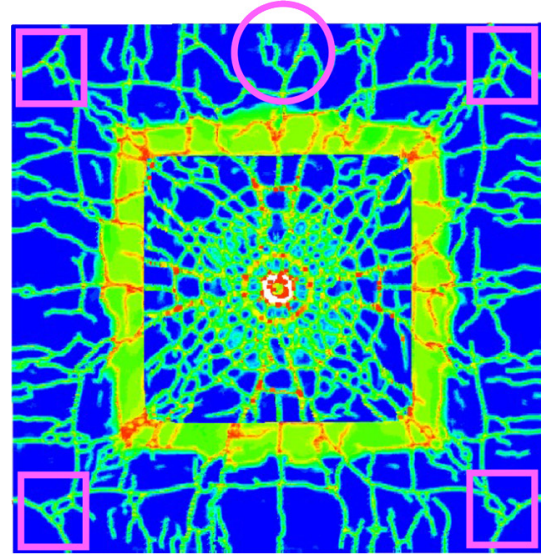

(a)

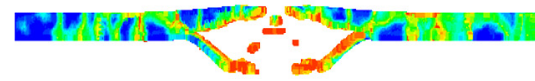

(c)

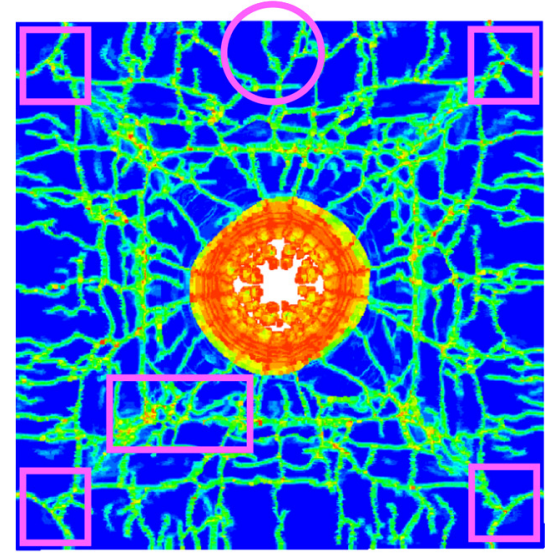

(b)

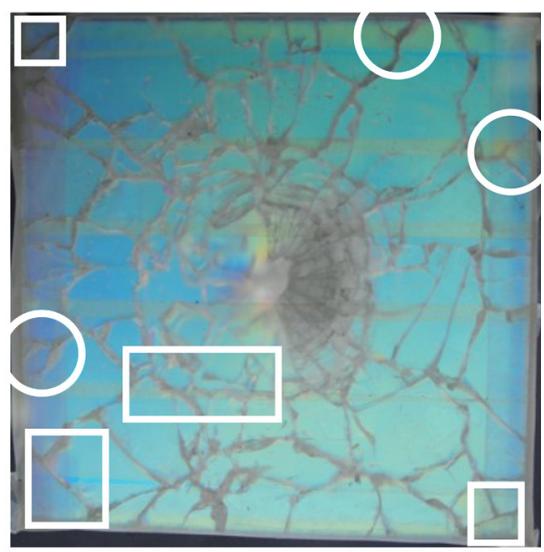

(d)

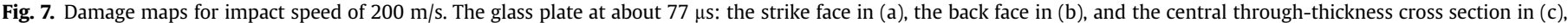
where displacements are enhanced by a factor of two, to better observe separation, and thickness direction is enlarged; experimental result shown in (d).

Some quadrant cracks still branch as they approach the boundaries (circular zones) and quite a few more cracks start from the boundaries and propagate inwards.

At this impact speed some plastic deformation is observed in the experiments in the polycarbonate layer, but no cracks. In the peridynamic solution, no damage (the only type allowed here would be crack formation, not plasticity) forms in the polycarbonate layer. Since the peridynamic model used in this work does not include plastic deformation, and additional means to reduce the energy in the system, such as dissipation through the boundary conditions, are not present in our model, we observe a somewhat larger amount of damage in the glass layer in the computations, compared with the experimental results.

\subsection{Trends with increasing projectile impact speed}

The projectile speed profiles are shown in Fig. 8. Shortly after the projectile hits the target, it rebounds, thus the negative sign of the projectile speed, for all three cases tested. This coincides with the experimental observation. The magnitude of the rebound velocity, however, is slightly different from the experimental tests: at $61 \mathrm{~m} / \mathrm{s}$ impact velocity the model gives a rebound speed of $13 \mathrm{~m} / \mathrm{s}$, while in the experiments it measures $3 \mathrm{~m} / \mathrm{s}$. At $100 \mathrm{~m} / \mathrm{s}$ impact speed, the computations show a rebound speed of $15 \mathrm{~m} / \mathrm{s}$, while experimentally it measures about $8 \mathrm{~m} / \mathrm{s}$. For the $200 \mathrm{~m} / \mathrm{s}$ case, the

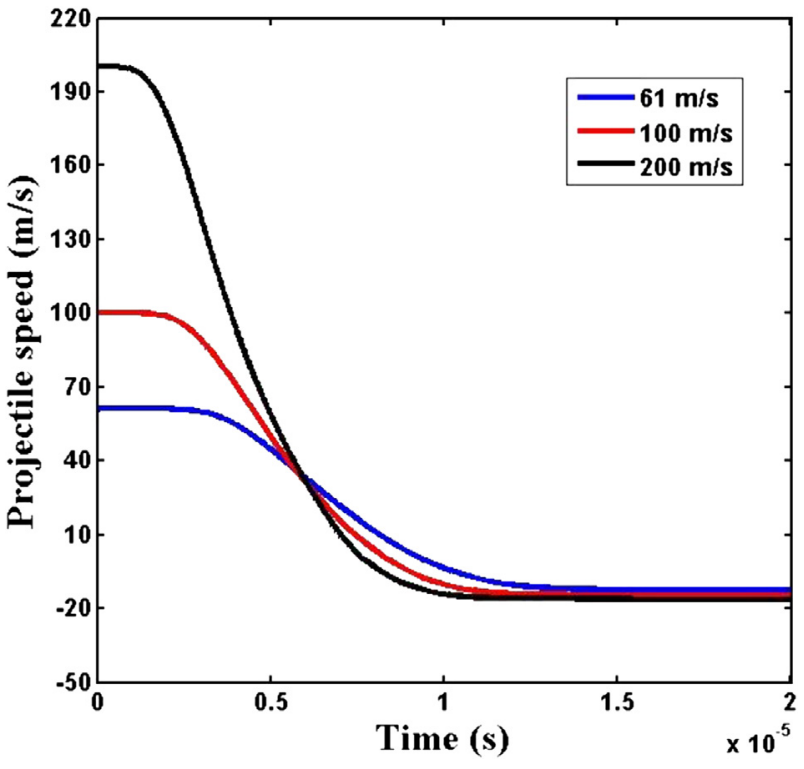

Fig. 8. Evolution of the projectile speed at different impact velocities. 
computations give $17 \mathrm{~m} / \mathrm{s}$ while the measured value is $33 \mathrm{~m} / \mathrm{s}$. Notice also that the projectile time-in-contact with the target, estimated from the velocity plots in Fig. 8 from the moment the speed starts to decrease until the moment the velocity becomes negative, is in the range of 6-7 $\mu$ s. These contact times are significantly higher than when small projectiles ( $1 \mathrm{~mm}$ diameter) impact a glass block, measured to be between 1 and $2 \mu$ s (see Ref. [7]). The difference is due to the bending deformation of the G-PC system used in our work and the larger projectile size (5.56 mm diameter). In Refs. [7], the glass block is thick enough $(2.5 \mathrm{~cm})$ so that the stress waves generated at the time of impact did not return to the impact site after reflecting from the edges of the glass block, during the contact time. In our case (thickness of $0.33 \mathrm{~cm}$ ), stress waves interact with the projectile.

While the trend observed in the experiments, of increased rebound velocity with an increasing impact speed, is matched by the peridynamic computations, two possible reasons are believed to contribute to the differences between the measured values and the peridynamic results:

- The use of a rigid model for the projectile: in reality the projectile deforms elasto-plastically and a complex transfer of energy between the deformable projectile and the PG-PC system takes place;

- Differences between the boundary conditions on the two-layer system that affect the bending structural response and thus the projectile rebound: in the experiments a metal frame and holding fixture are used, while in the simulations these conditions are replaced by imposed zero out-of-plane displacements.

Nevertheless, the fact that the trend of increasing rebound speed with an increased impact speed is well captured by this simple peridynamic model and that the computed values are in a similar range with those measured experimentally are very encouraging.

\subsection{The computed time-evolution of damage}

In what follows we analyze the evolution of damage in the glass layer in the first $25 \mu \mathrm{s}$ from impact. The $61 \mathrm{~m} / \mathrm{s}$ impact speed case is discussed here.

From the results shown in Fig. 9 for the median cross-section through the thickness, we observe that the cracks start from the
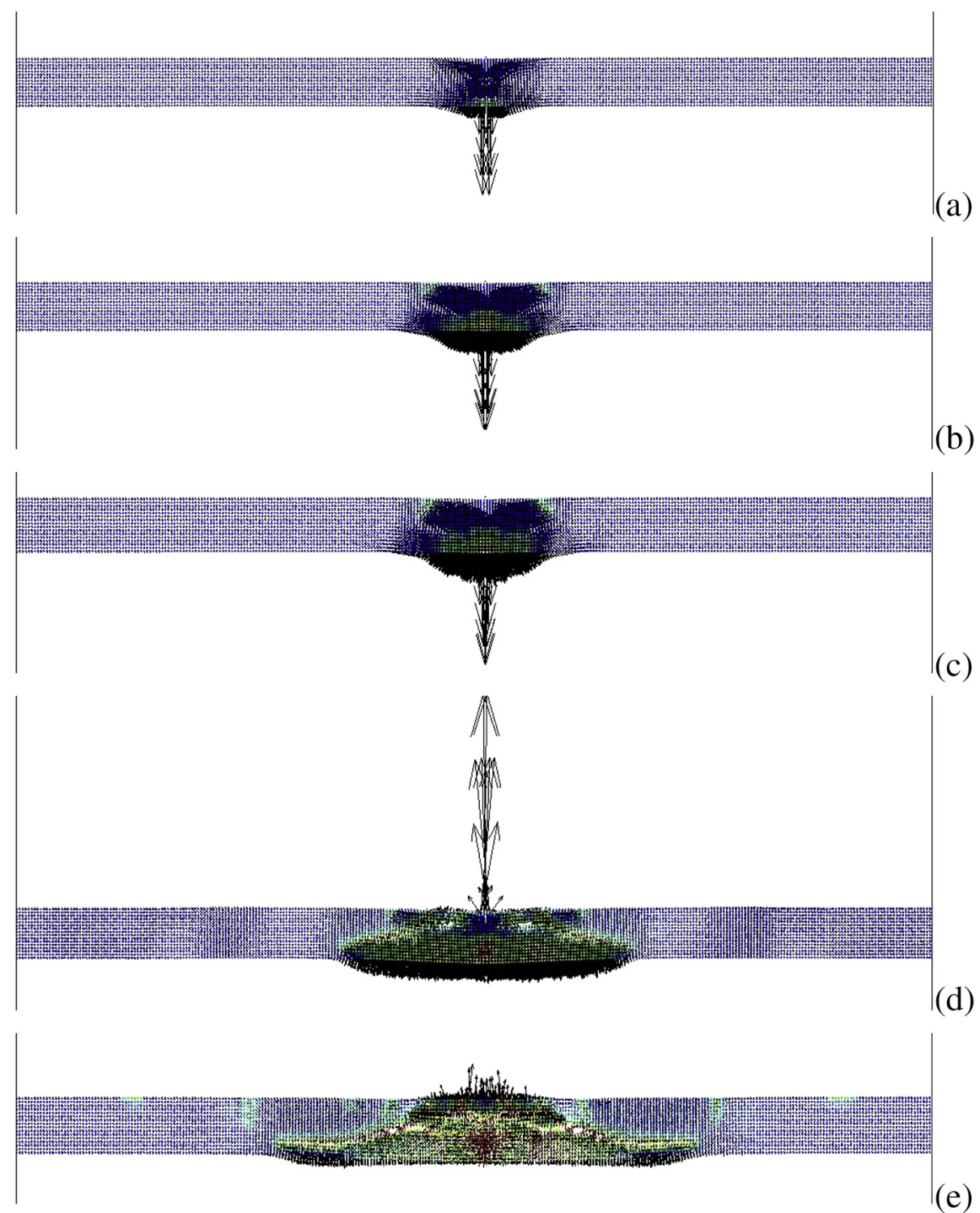

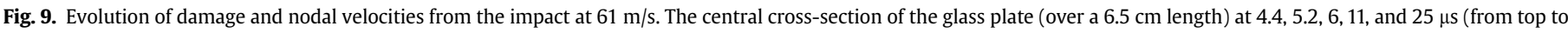
bottom, see movie 1 in supplementary material). Nodal velocities are plotted using black arrows (quiver plot) over the damage maps. 
bottom face of the glass plate. These initial cracks are radial cracks in the form of an eight-spoke wheel. They propagate radially but also grow upwards through the thickness. This confirms the results from molecular dynamic simulations presented in Ref. [12]. The cracking from bottom to top has also been observed in computations of high velocity impact on a seven-layer glass system (see Ref. [22]). The first cracks on the top face form in the shape of a ring crack (see Figs. 9 and 10), around 5.2 $\mu$ s from the moment of impact.

Supplementary video related to this article can be found at http://dx.doi.org/10.1016/j.ijimpeng.2013.07.001.

Driven by the stress waves, the radial cracks from the bottom and the ring crack on the top face move towards one another and deflect from each other, before they coalesce. The crack interactions and the continuing propagation and reflection of stress waves in the system then lead to the formation of other ring cracks on the top face, both inside and outside of the original ring crack of the top face. A Hertzian cone-crack is fully formed around $11 \mu \mathrm{s}$. The reflections from the boundaries (the frame region) further complicate the evolution of the damage, as can be seen from Fig. 10. The presence of the boundary conditions that approximate the existence of the metal frame holding fixture generates straight cracks that propagate on the top face only. We call these "frame cracks". These are mainly the features that are located where the frame opening is. In the simulations, since the top surface of the glass is held in place, surface cracks (chips and flakes) are produced, while the constraint on the displacements of the bottom face of the PC layer is responsible for inducing the through thickness cracks. In the experiments, these "frame cracks" happen only where the PC and the glass happen to come in contact with the frame. In the experiments, the clamps are on the outer edges of the frame, not on the inner edges and due to the impact the glass plate loses contact with the top metal frame. If direct contact between the PC plate and the inner part of the frame takes place then that gives rise to conditions that initiate cracks that trace portions of the frame opening (see cracks in the rectangular contours on the pictures in Fig. 2).

The computational results show that on the top face, wave interactions create conditions for glass chips to form in the middle of the inner bounds of the metal frame region. These surface cracks continue to grow as they propagate both towards the boundaries of the sample and along the frame cracks. Radial cracks grow and arrest before reaching the frame cracks, but eventually continue towards them, and cross them merging and getting ahead of the surface cracks. Radial cracks are primarily driven along the bottom face and they cut through to the top face as they propagate, but not always perpendicular to the faces. The non-planar radial cracks and their tortuosity is a direct consequence of interactions between the variety of stress waves reflecting from the boundaries and the propagating crack.

The spokes in the 45-degree direction eventually arrest due to interactions with reflected waves, and only the so-called quadrant cracks (the horizontal and vertical spokes) continue to propagate, but their paths and speed of propagation are also perturbed by interactions with stress waves. Eventually, new diagonal cracks appear near the corners of the opening region and on the top face first, initiated by the interaction of waves that reflect from the sides of the "frame opening". These cracks grow in both directions (towards the corners and the center) in the in-plane direction, and also grow through the thickness to give rise to diagonal cracks on the bottom face. At that stage, the diagonal cracks on the bottom face merge with the original diagonal cracks that started at the center.

The vector plots of the nodal velocity (see Figs. 9 and 10) superposed onto the damage index plots clearly indicate the propagation of the surface waves (Rayleigh waves) on the top and bottom faces of the glass plate. The complex interaction among longitudinal, shear, and surface waves, with the reflected waves from the boundaries and the polycarbonate interface, depict a complex picture of how damage is generated in the two-layer system at this impact velocity. Plans for the future include experimental setups capable of recording the evolution of damage in a two-layer system.

To further analyze the influence of boundary conditions on our simulation results, in the next section we present experiments and simulations for a setup that does not involve a frame.
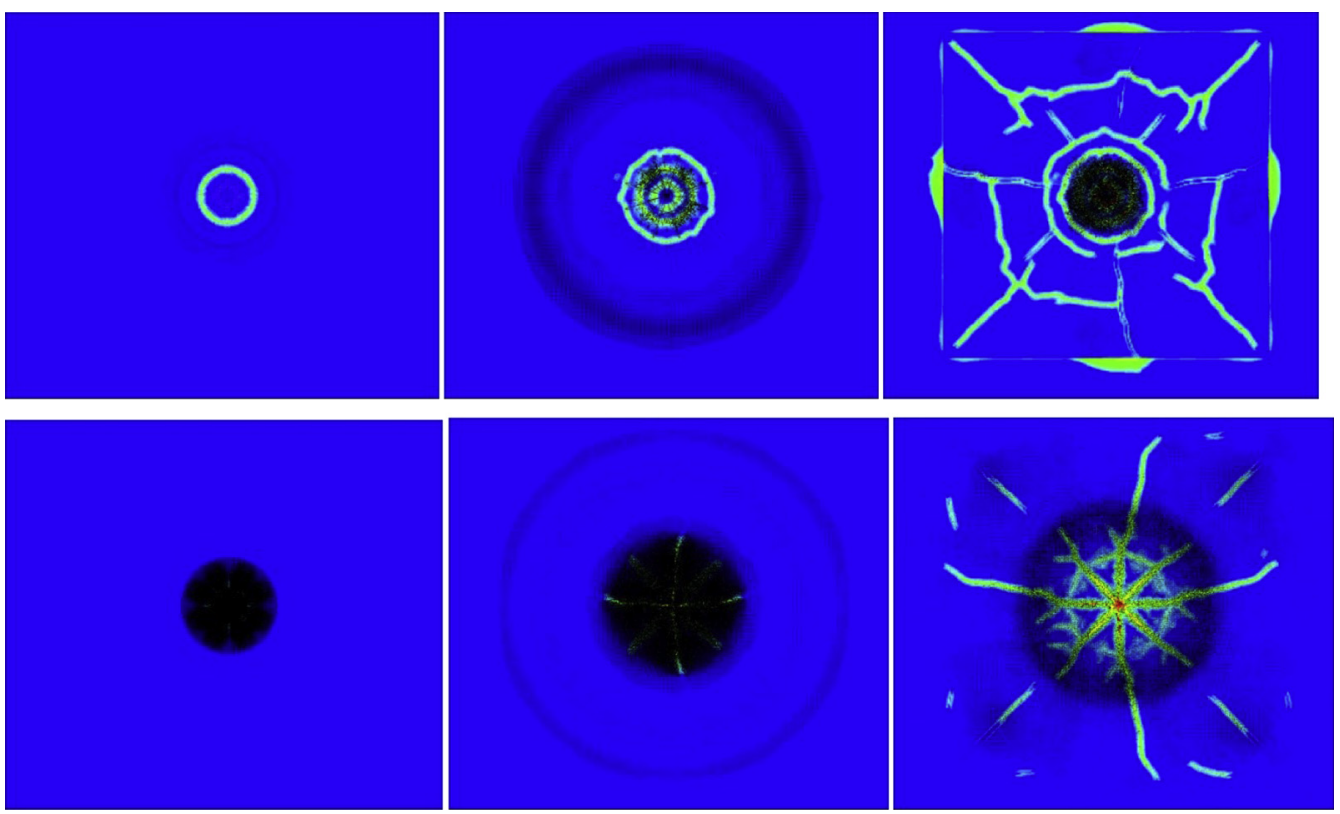

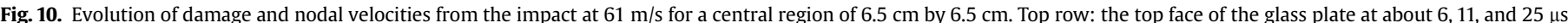

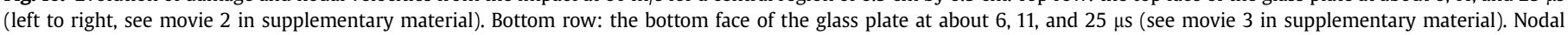
velocities are plotted using black arrows (quiver plot) over the damage maps. 


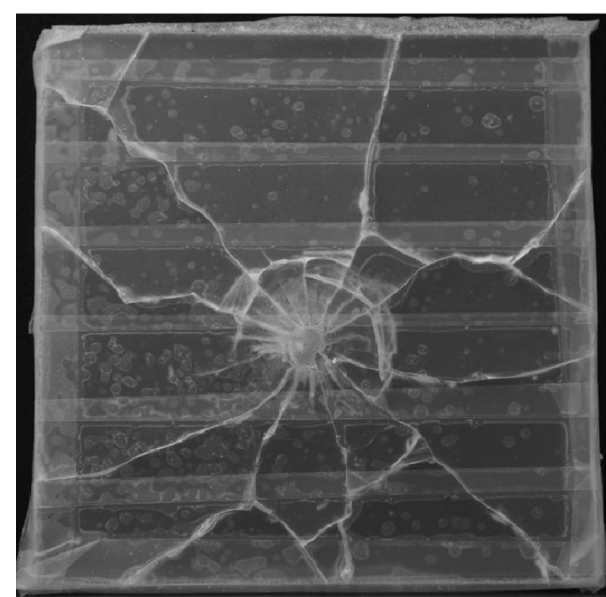

$[58 \mathrm{~m} / \mathrm{s}]$

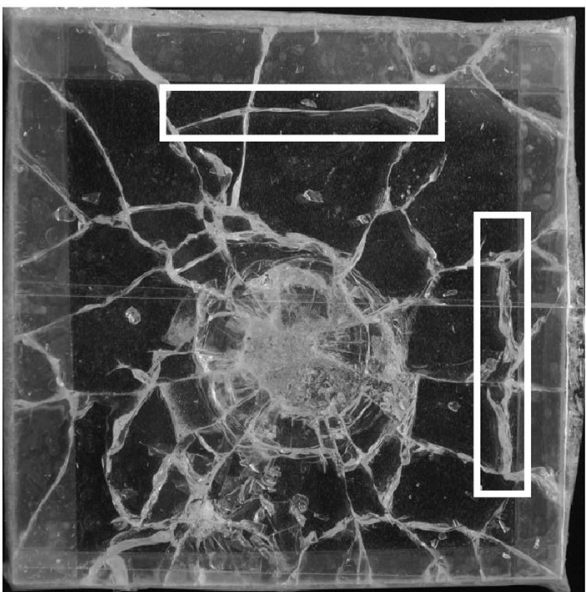

$[150 \mathrm{~m} / \mathrm{s}]$

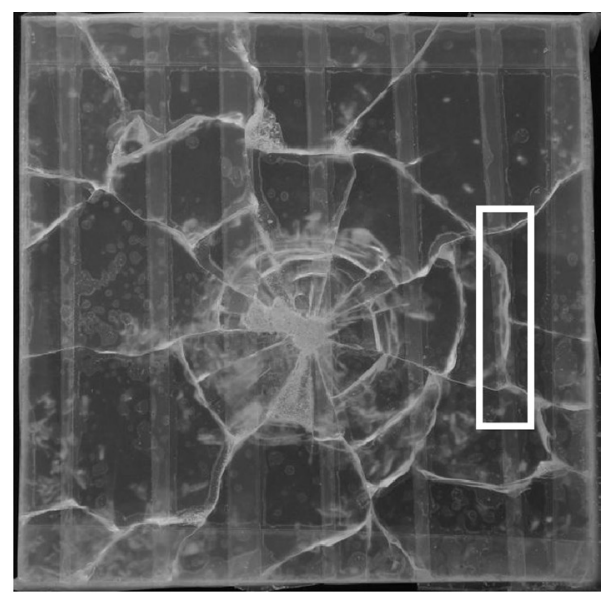

$[100 \mathrm{~m} / \mathrm{s}]$

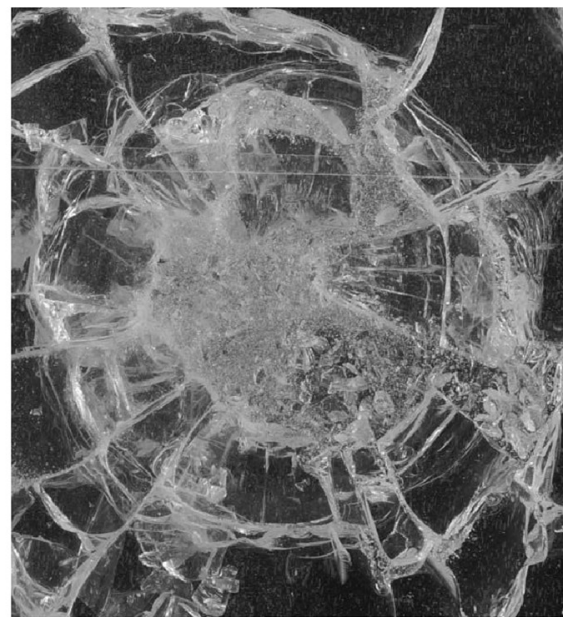

$[150 \mathrm{~m} / \mathrm{s}]$ zoom in near the impact

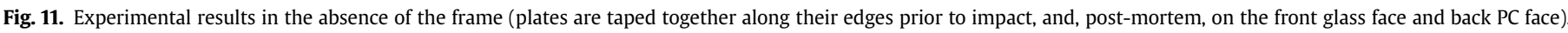
View from the impact face. At the higher impact speeds some glass fragments from the glass back face have moved and are stuck between the plates.

\section{Analysis of influence of boundary conditions: experiments and peridynamic simulations}

We conduct a new set of experiments that does not involve a frame. In this case the plates are suspended (by tape), and, to be able to recover glass fragments in the experiments, the two plates are taped together along their boundaries only. The front face of the glass plate is taped over only after the experiment to prevent further loss of fragments from handling. The computational model for these tests has no boundary conditions (mimicking the suspended plates from the experiment), so the two plates are in contact but otherwise free. We do not simulate the taping of the two plates along their edges used in experiments. The new results, together with the original ones, should provide a better understanding of the role and influence of boundary conditions in dynamic brittle fracture of glass in multilayered configurations.

We again focus on the types and shapes of the cracks observed since they are an indication of the dynamics of the fracture process. A kink and tilt in a crack at a certain location in the plate is caused by interaction with the stress waves reflected from the boundaries or the material surfaces. Obtaining such features in the simulations at similar locations as in the experiments indicates that the computed cracks move with the same speeds as in the experiments. In crack branching events, the authors have previously published results (see Refs. $[17,18,21])$ that demonstrated that, in the presence of stress waves, a horizon size of about $1 \mathrm{~mm}$ or smaller results in matching observed crack propagation speeds, while in cases in which the propagation of the crack is not influenced by incoming stress waves reflected from the boundaries, any horizon size gives the observed propagation speed.

Due to the new experimental conditions (suspended plates), the projectile does not hit the center of the plate, but about $0.7-1 \mathrm{~cm}$ closer to one of the boundaries of the plate (see Fig. 11). In the computations we use the approximate impact location measured from the post-mortem samples. The experimentally measured impact velocities just before impact are: $58 \mathrm{~m} / \mathrm{s}, 100 \mathrm{~m} / \mathrm{s}$, and $150 \mathrm{~m} / \mathrm{s}$. The PC plate is not damaged at these impact speeds.

The following differences between the experimental results with the frame and without the frame can be observed (compare Figs. 2 and 11):

- The cracks present around the frame opening in Fig. 2 (see rectangular contours) are now absent at the lower impact speed, confirming the conclusion about the origin of these cracks discussed before. At the higher speeds, however, cracks that run near the boundaries and parallel to them are observed (see rectangular contours in Fig. 11). These cracks are likely induced by stress waves reflected 

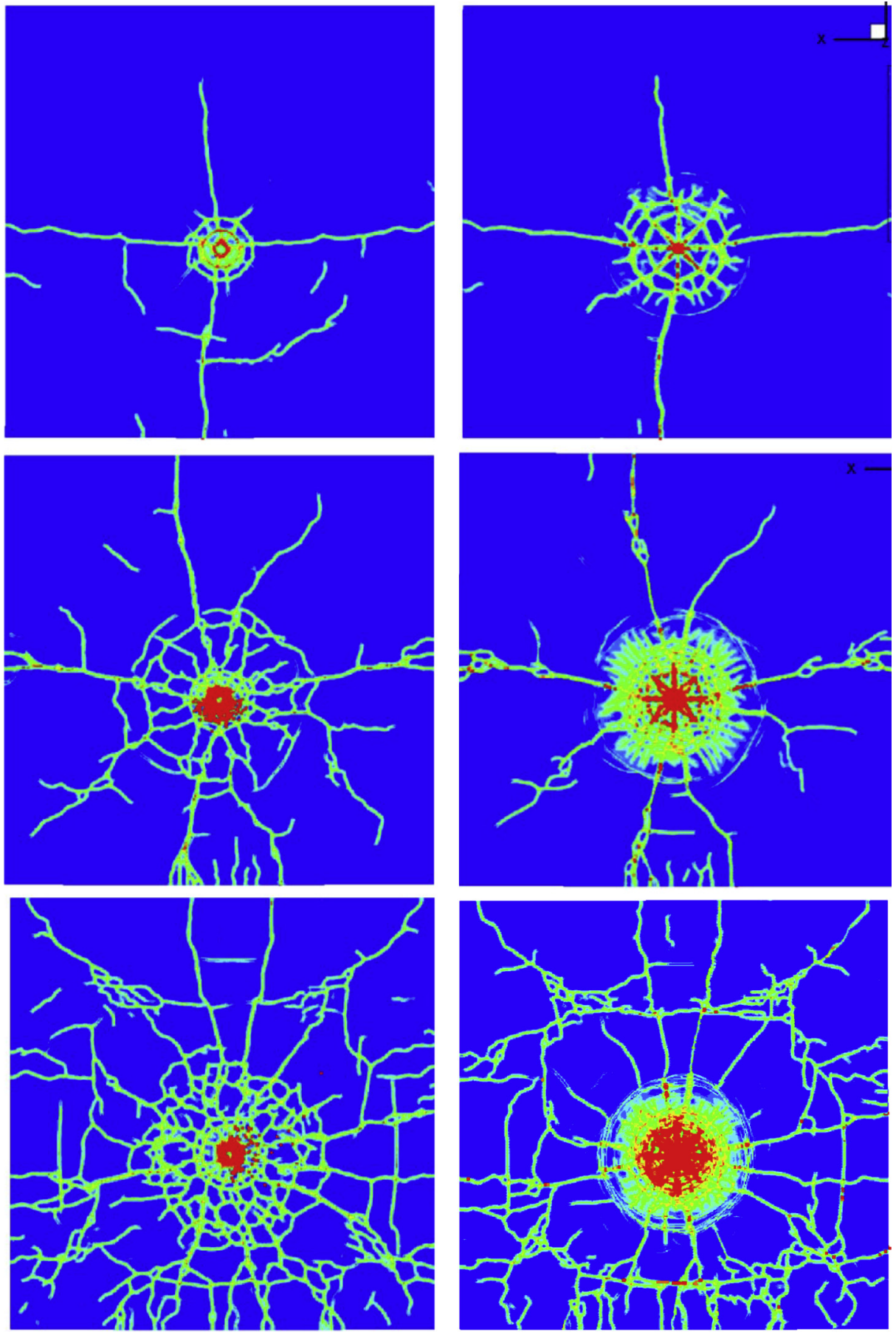

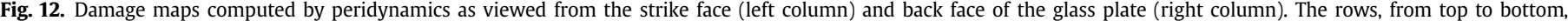
correspond to the impact speeds of $58 \mathrm{~m} / \mathrm{s}, 100 \mathrm{~m} / \mathrm{s}$, and $150 \mathrm{~m} / \mathrm{s}$ at about $100 \mu \mathrm{s}$ after impact, respectively.

from the edge boundaries. The location of these cracks, relative to the impact location, appears to reproduce the asymmetry of the impact location relative to the center of the plate.

- Corner cracks are not present in the absence of the frame, leading us to conclude that these cracks were induced by stress waves generated from the interaction between the plates and the frame.

- The branching cracks that were near the boundaries in Fig. 2, are still present and their prevalence increases with the increased impact speed.
- There are only a few cracks that emanate from a boundary and would not connect with other radial cracks emanating from the center. Thus, many boundary cracks are a result of the presence of the confining frame.

- A set of very fine circumferential "cracks"1 around the coarse circumferential cracks in the Hertzian cone region (about $1.5-2 \mathrm{~cm}$ away from the impact point) are clearly visible in the $100 \mathrm{~m} / \mathrm{s}$ and $150 \mathrm{~m} / \mathrm{s}$ cases. These "cracks" do not feel to

\footnotetext{
1 The reason for the quotation marks will become clear in what follows
} 
the touch on the top glass surface (see also the zoom in Fig. 11).

- Some of the glass fragments from the Hertzian cone region on the back face of the glass plate have shifted from their original locations and are trapped between the two plates.

The total simulation time is $100 \mu \mathrm{s}$, and the initial location of the rigid projectile is just above the glass plate and therefore impact happens after the first few time steps. The peridynamic computations are performed using the parameters as before: horizon size $\delta=1 \mathrm{~mm}$ and a grid spacing $\Delta \mathrm{x}$ defined by $\mathrm{m}=\delta / \Delta \mathrm{x}=4$; the time step is still $27.5 \mathrm{~ns}$.

The results in terms of the damage maps, at about $100 \mu \mathrm{s}$ after impact, viewed from the strike side and from the back-face of the glass plate are shown in Fig. 12. While the bulk of crack growth has ended by end-time of these simulations, a few cracks are still growing, and more cracks would form if we had the PC and glass plates taped along their edges in our computations, due to the elastic rebound of the PC plate onto the cracked glass plate. In experiments, the rebound of the PC plate is likely the cause for which we now see less cracks in the simulations than in the experiment with the $58 \mathrm{~m} / \mathrm{s}$ test conditions. The elastic rebound is not captured in these simulations due to the absence of the edge taping between the plates, and the short simulation time. Moreover, in the experiments, the forced contact at the ends of the plates due to the edge-tape certainly increases wave reflections and elastic energy transfer between the plates, possibly leading to extra damage compared to a case with no edge-tape. In experiments, the tape is needed so that the glass fragments are recovered. The only difference in the boundary conditions used for the computational model compared to the experimental conditions is the absence of edge tape that keeps them together.

At the higher impact velocity, the computational results match very closely the observed damage patterns.

For the $100 \mathrm{~m} / \mathrm{s}$ impact speed case, damage progression happens as follows: the impact creates ring cracks on the top surface (due to deformation at the impact site) while on the bottom, stress waves create hoop stresses that result in radial cracks. These two damage fronts interact with each other in forming a shallow Hertzian cone, which is deflected into a transverse crack by stress waves. The transverse crack splits the glass parallel to the strike plane. Some of the radial cracks growing on the bottom face eventually arrest, and only a few continue to grow.

A particular "crack" system seen in both the experiments and the computations is the set of fine circumferential "cracks" surrounding the Hertzian cone damage area on the bottom face of the glass (see Fig. 12). These cracks are not complete circumferential cracks, meaning they appear late in the damage process (fact confirmed by our simulations), and are much finer than other circumferential cracks seen on either the top or the bottom surfaces. It is interesting to notice that the damage lines on the bottom face of the glass plate obtained by the peridynamic model show damage levels of about $20-30 \%$, indicating that these are not fully developed crack surfaces on the bottom face of the glass. Analysis of the computational results through a transverse cross-section near the impact point reveals that the fine lines are the signature of "steps" or roughness from the transverse crack that splits the glass parallel to the strike face (see Fig. 13). These ridges where found on the fragments from the experiments also, and, as in our computations, the bottom glass surface, where these ridges appear, is intact. The conclusion is that these fine features appear on the surface of the transverse crack that grows from the deflected Hertz cone crack, and as the layer is very thin, stress waves bounce rapidly from the bottom of the glass plate and create these micron-size

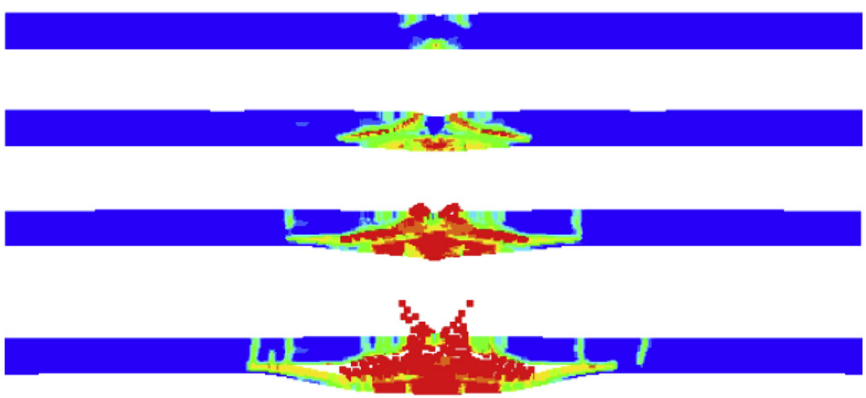

Fig. 13. Damage progression seen along a cross-section near the impact location for the $100 \mathrm{~m} / \mathrm{s}$ impact speed case. Observe the formation of the transverse cracks (parallel with the strike face of the plate). Waves reflected from the boundaries ripple over this region and create roughness which shows as the fine circumferential "cracks" observed in Fig. 11.

light-reflecting ridges. A more detailed analysis of these interesting damage features will be provided in a future publication.

While the impact problem on the two-layer system is significantly more difficult than the dynamic branching of a single crack treated in Refs. [17,18,21], the results shown here demonstrate how peridynamics can aid in understanding the dynamics of the process of fracture and damage in difficult scenarios.

\section{Conclusions}

This paper presents experimental and numerical simulation results of the impact damage in a thin glass layer with a thin polycarbonate backing subject to impact from a small spherical projectile at different speeds. Significant changes in the damage patterns that develop in the glass plate at three different impact speeds take place in the experimental tests. These changes are predicted by the peridynamic model in both the case with a metal frame holding the two plates together, or without the metal frame. Some very specific damage and crack features observed in experiments are, remarkably, reproduced by the computational model, at about the same location as in the experiments. This allows us to conclude that, the cracks computed by the peridynamic model move at a similar speed as those in the experiments.

The numerical results are obtained here using the simplest peridynamic model for micro-elastic brittle materials. We can conclude that at these levels of loading, modeling the glass plate as a linear micro-elastic brittle material is sufficient to explain the damage structure from impact in the thin glass plate with a thin polycarbonate backing plate. The simplified boundary conditions used in the computational model is the most important factor for the differences between the experiments and the computations in this problem.

In both the experiments and the peridynamic simulations, ejection of the projectile from the target is observed, and the trend of increasing rebound velocity with increased impact speed observed in experiments is reproduced by the peridynamic model. The computational results allow for an understanding of the complex evolution of damage in the glass layer induced by stress waves propagation and reflections. In the peridynamic model, no special crack initiation and propagation criteria and/or special meshing techniques are necessary.

The results presented here lead us to conclude that, for predicting dynamic brittle fracture, determining factors are: the elastic properties of the material (since stress waves play such an important role in the evolution of fracture), the fracture energy of the material, and a model that correctly traces the bouncing of stress waves from surfaces and interfaces, and which allows for 
unrestricted crack evolution and feedback-loop interaction between advancing crack fronts and stress waves. The peridynamic model used here, the simplest one can think of, can be extended to incorporate, for example, effects such as toughening of glass (via compressive residual stresses at the glass surface).

Plans for the future also include more accurate description of the holding fixtures/boundary conditions. In dynamic fracture and damage of brittle materials, boundary conditions are important factors that influence stress waves propagation in the system, that, in turn, control the evolution of failure fronts and the growth and propagation of damage in the system. Experimental setups monitoring the time-evolution of damage and comparisons with the damage progression obtained from the peridynamic models are also planned.

\section{Acknowledgment}

The authors are thankful for the financial support offered through research contracts between UNL and the ARO (Dr. Larry Russell), and ARL (project coordinators Dr. C.F. Yen), ARO award number 58450 EG. The last author acknowledges partial support from Callahan Research of New Zeeland (project coordinator Dr. Biswajit Banerjee). The computations in this paper have been completed using facilities at the Holland Computing Center of the University of Nebraska-Lincoln.

\section{References}

[1] Knight CG, Swain MV, Chaudhri MM. Impacts of small steel spheres on glass surfaces. J Mater Sci 1977;12:1573-86.

[2] Ball A, Mckenzie HW. On the low velocity impact behaviour of glass plates. J Phys IV 1994;4:783-8.

[3] Walley SM, Field JE. The contribution of the Cavendish laboratory to the understanding of solid particle erosion mechanisms. Wear 2005;258:552-66.

[4] Field JE. Dynamic fracture: its study and application. Contemp Phys 1971;12: $1-31$.

[5] Field JE. Investigation of the impact performance of various glass and ceramic systems. Cambridge, U.K.: European Research Office of the US Army; 1988. Report No. R\&D 5087-MS-01. p. 14-5.
[6] Field JE, Sun O. Townsend ballistic impact of ceramics. Inst Phys Conf Ser 1989;102:387-93.

[7] Chaudhri MM, Walley SM. Damage to glass surfaces by the impact of small glass and steel spheres. Philos Mag A 1978;37:153-65.

8] Bless SJ, Chen T. Impact damage in layered glass. Int J Fract 2010;162:151-8.

[9] Timmel M, Kolling S, Osterrieder P, DuBois PA. A finite element model for impact simulation with laminated glass. Int J Impact Eng 2007;34:1465-78.

[10] Ismail J, Zairi F, Nait-Abdelaziz M, Azari Z. How cracks affect the contact characteristics during impact of solid particles on glass surface: a computational study using anisotropic continuum damage mechanics. Int J Impact Eng 2012;40-41:10-5.

[11] Pyttel T, Liebertz H, Cai J. Failure criterion for laminated glass under impact loading and its application in finite element simulation. Int J Impact Eng 2011;38:252-63.

[12] Holmstrom E, Samela J, Nordlund K. Atomistic simulations of fracture in sillica glass through hypervelocity impact. Europhys Lett 2011;96. 16005-pp.116005-p.5.

[13] Ravi-Chandar K. Dynamic fracture. Amsterdam: Elsevier; 2004. p. 208-16.

[14] Silling SA. Fragmentation modeling with EMU. Albuquerque, NM: Sandia National Laboratories; 2005. Technical report. p. 6-7.

[15] Bobaru F, Ha YD. Adaptive refinement and multiscale modeling in 2D peridynamics. Int J Multiscale Comput Eng 2011;9:635-60.

[16] Silling SA. Reformulation of elasticity theory for discontinuities and longrange forces. J Mech Phys Solids 2000;48:175-209.

[17] Ha YD, Bobaru F. Studies of dynamic crack propagation and crack branching with peridynamics. Int J Fract 2010;162:229-44.

[18] Ha YD, Bobaru F. Characteristics of dynamic brittle fracture captured with peridynamics. Eng Fract Mech 2011;78:1156-68.

[19] Hu W, Ha YD, Bobaru F. Modeling dynamic fracture and damage in fiberreinforced composites with peridynamics. Int $\mathrm{J}$ Multiscale Comput Eng 2011;9:707-26

[20] Hu W, Ha YD, Bobaru F. Peridynamic simulations of dynamic fracture in unidirectional fiber-reinforced composites. Comput Meth Appl Mech Eng 2012;217-220:247-61.

[21] Bobaru F, Hu W. The meaning, selection, and use of the peridynamic horizon. Int J Fract 2012;176:215-22.

[22] Bobaru F, Ha YD, Hu W. Damage progression from impact in multilayered glass modeled with peridynamics. Cen Eur J Eng 2012;2:551-61.

[23] Seleson P, Parks M. On the role of the influence function in the peridynamic theory. Int J Multiscale Comput Eng 2011;9:689-706.

[24] Weckner O, Silling SA. Determination of nonlocal constitutive equations from phonon dispersion relations. Int J Multiscale Comput Eng 2011;9:623-34.

[25] Silling SA, Askari E. A meshfree method based on peridynamic model of solid mechanics. Comput Struct 2005;83:1526-35.

[26] Silling SA, Epton M, Weckner O, Xu J, Askari E. Peridynamic states and constitutive modeling. J Elasticity 2007;88:151-84.

[27] Hu W. Peridynamic models for dynamic brittle fracture. Lincoln, NE: University of Nebraksa-Lincoln; 2012. Ph.D. dissertation. 